\title{
Case Report: Vibrio cholerae Biliary Tract Infections in Two North Africans in France
}

\author{
Philippe Parola, ${ }^{1,2 \star}$ Hussein Anani, ${ }^{1,2}$ Carole Eldin, ${ }^{1,2}$ Gregory Dubourg, ${ }^{2,3}$ Jean-Christophe Lagier, ${ }^{2,3}$ Anthony Levasseur, ${ }^{2,3}$ \\ Jean-Paul Casalta, ${ }^{2}$ Didier Raoult, ${ }^{2,3}$ and Pierre-Edouard Fournier ${ }^{1,2}$

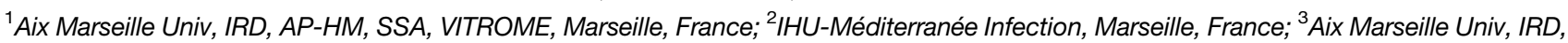 \\ AP-HM, MEPHI, Marseille, France
}

\begin{abstract}
The origin of a cholera outbreak may be unclear, as recently in Algeria. In two patients from North Africa, Vibrio cholerae was isolated in the context of hepatobiliary tract infections without any known outbreak. Gallbladder and asymptomatic long-term carriers might play a role in the emergence of cholera.
\end{abstract}

Cholera is an acute, watery diarrheal illness caused by the bacterium Vibrio cholerae. It remains a public health issue in many parts of the world, with millions of cases and around 100,000 deaths annually. ${ }^{1}$ The environmental niches of $V$. cholerae include saline coastal waters, rivers, and estuaries, often in association with crustacean zooplankton. Thus far, 210 serogroups of $V$. cholerae have been described, among which those secreting the cholera toxin can cause outbreaks. Vibrio cholerae serogroup 01 biotype El Tor is the cause of the current pandemic that has been ongoing since the 1960s. ${ }^{1}$ Major outbreaks are currently ongoing, mainly in sub-Saharan Africa and in Hispaniola. ${ }^{1}$ Serotype O139, which emerged in the 1990s and spanned most of Asia, is now rarely isolated. ${ }^{1}$

In August 2018, the Algerian health authorities disclosed a cholera outbreak in the northern part of Algeria, almost 40 years after the last epidemic in the country. ${ }^{2}$ In early September 2018 , more than 200 patients with cholera-like symptoms had been hospitalized, leading to two deaths. Cases were reported in seven wilayas (provinces), and $V$. cholerae was identified in the city of Blida, in the Bani Azza river that extends to the beach in the Algiers area. Vibrio cholerae O1 biotype El Tor serotype Ogawa was confirmed in 83 cases at the Pasteur Institute in Algiers. ${ }^{2}$

The most recent information suggests that the outbreak is now over. ${ }^{1}$ However, the origin of the emergence of this epidemic is unclear. Recent genomic works suggest that, apparently, epidemic clones do not implant themselves in endemic form outside Asian foci. ${ }^{1}$ Human movement from Asia would have the greatest impact for the spread and persistence of $V$. cholerae, rather than the reemergence of a local lineage of $V$. cholerae. ${ }^{1}$ We report here two cases from Algeria and Tunisia, in which $V$. cholerae was isolated in the absence of any watery diarrheal symptoms or any known cluster or outbreak of cholera.

\section{CASE REPORTS}

Case 1: A 21-year-old woman with no specific medical history underwent a cholecystectomy under laparoscopy in June 2017 in Oran, Algeria, as the result of biliary colic. A T-shaped tube was inserted intraoperatively to deal with biliary leak following the cholecystectomy. Analysis of the gall bladder revealed signs of chronic cholecystitis. Forty-eight hours after surgery, she became icteric with abdominal pain. Another surgical procedure found a postoperative complication with a laceration in the common bile duct and the common hepatic duct which

*Address correspondence to Philippe Parola, VITROME, Institut Hospitalo-Universitaire Méditerranée Infection, 19-21 Blvd. Jean Moulin, Marseille 13005, France. E-mail: philippe.parola@univ-amu.fr extended to the convergence. This laceration was responsible for a chronic biliary peritoneal effusion, icterus (total bilirubinaemia: $45 \mu \mathrm{mol} / \mathrm{L}$ ), and hepatic cytolysis (aspartate transaminase: $217 \mathrm{UI} / \mathrm{L}$ and Alanine transaminase: $283 \mathrm{UI} / \mathrm{L})$. She was operated upon in September 2017 in Marseille, France, to repair the common bile duct and was transferred to a ward with Jackson-Pratt drains. Forty-eight hours after this surgery, she became febrile. Biliary fluids were collected from the drains and sent for microbiological analysis. After 24 hours, $V$. cholerae was identified in the culture (strain G 1596). One sample also yielded the presence of Morganella morganii. The patient was treated with doxycycline and ciprofloxacin for 15 days and remained healthy. No family member living with the patient carried any Vibrio.

Case 2: A 69-year-old Tunisian patient who has been living in France for 30 years, and who made frequent trips to his native country every year, was hospitalized in July 2018 near Marseille, France. His medical history included gastric adenocarcinoma in 2012, which was treated with gastrectomy and chemotherapy, with a final check in 2016, and a cholecystectomy. He presented on July 12, 2018 with epigastric pain, transient diarrhea, and no fever. The blood bioassay showed elevated liver transaminases, cholestasis (aspartate transaminase $121 \mathrm{UI} / \mathrm{L}$, alanine transaminase $142 \mathrm{UI} / \mathrm{L}$, alcaline phosphatase $479 \mathrm{UI} / \mathrm{L}$, gammaglutamyl transferase $424 \mathrm{UI} / \mathrm{L})$, hyperbilirubinaemia $(26 \mu \mathrm{mol} / \mathrm{L})$, and polymorphonuclear leukocytosis (11 Giga/L). A gastric endoscopy was normal. He received empirical treatment through amoxiclav plus ciprofloxacin. When three blood cultures collected on July 19, 2018 isolated V. cholerae (strain P8118), he received empirical treatment with ceftriaxone and doxycycline and was referred to our center. No fever or diarrhea was observed. A computerized tomography (CT) scan on July 26, 2018 showed hypotonia of the intrahepatic bile ducts and the main bile duct, with enhancement of the wall of the common bile duct, evoking choledochitis, multiple and bi-lobar hypodense and hypo-elevated intrahepatic formations, with an enhanced appearance at the portal wall, suggesting abscess. Stool cultures performed during the antibiotic treatment remained negative. He recovered in 1 week.

\section{GENOME SEQUENCING}

The genomes of the strains of both patients were sequenced using a MiSeq sequencer and the mate pair strategy (Illumina, San Diego, CA). The Algerian strain G1596 exhibited a

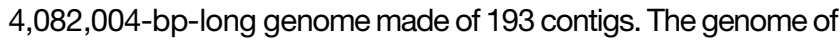
the Tunisian strain P8118 was 3,692,732-bp long and was made of 1,253 contigs. Based on core genome analysis, these two strains were clustered in a $V$. cholerae group named pandemic sister group $^{3}$ (Figure 1). Members of this group have previously been 
demonstrated to cause outbreaks. ${ }^{3,4}$ Strain P8118 exhibited a Vibrio pathogenicity island 1 similar to that of strain 2012Env-9, a representative of the pandemic sister group. ${ }^{3,4}$ By contrast, the genome from strain G1596 did not contain this pathogenicity island but multiple toxin-encoding genes (relE2, higB-2, and higA-2).

\section{DISCUSSION}

Interestingly, our two cases link the hepatobiliary tract and V. cholerae. In 1967, the observation of 43 convalescent cholera patients, including two asymptomatic carriers who underwent stool examination and duodenal aspiration, suggested that the gallbladder may harbor vibrios in convalescent cholera. ${ }^{5}$ The same year, the first known long-term carrier of cholera known as Cholera Dolores was described in the Philippines. ${ }^{6}$ Duodenal intubation suggested that the vibrios were harbored in the gallbladder or somewhere in the biliary tract. Since then, one case of cholera with watery diarrhea associated with acute acalculous cholecystitis was reported in 1995 with V. cholerae O1 El Tor isolated in the fluid, and a "milky" sediment was found within the gallbladder after cholecystectomy. ${ }^{7}$ In 1996, a patient from Bolivia developed symptoms of acute cholecystitis and his bile culture grew $V$. cholerae $01 \mathrm{El}$ tor. $^{8}$ In addition, non-01 strains have been isolated from patients with several infections and from the gallbladder, ${ }^{9-11}$ as reported in our patient from Algeria.

Here, it was found that strains P8118 and G1596, both harboring virulence genes that suggest their pathogenic potential, are related to pandemic $V$. cholerae strains, which may constitute a reservoir of cholera outbreak in North Africa. However, the existence of such carriers and their role in the recurrence and transmission of cholera deserve further investigation. Another issue is that $V$. cholerae cholera agents are reputed to be noninvasive, with bacteremia being an apparently rare event. Three blood cultures were however positive in our Tunisian patient. Interestingly, the first case of a septicemia due to V. cholerae 0139

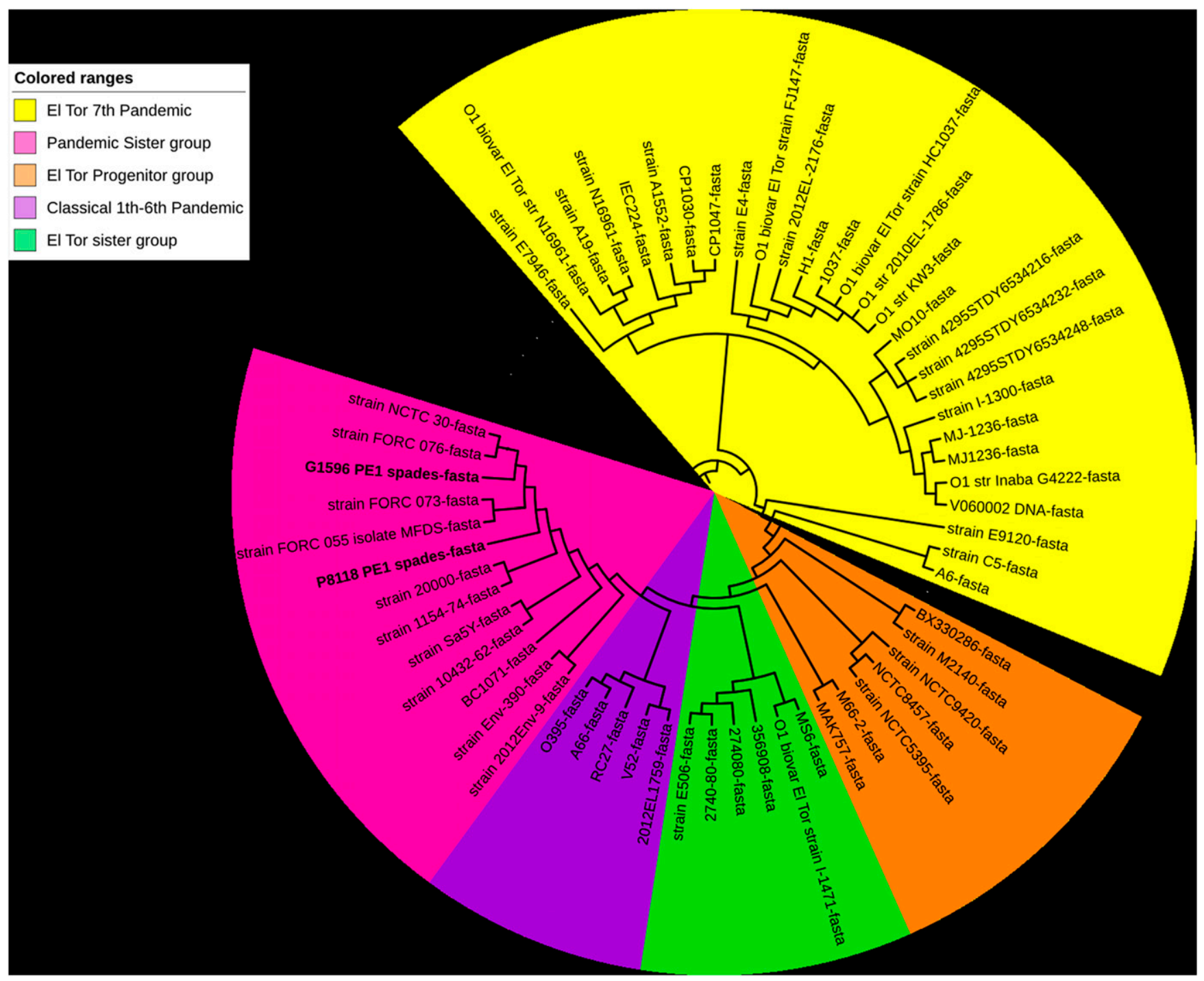

FIGURE 1. Phylogenetic tree showing the core genome-based phylogeny between studied Vibrio cholerae strains. Based on the literature, we selected 63 strains belonging to pandemic groups 3 and 4, and their genomes were downloaded from GenBank. The 65 genomes of Vibrio sp. strains were annotated with Prokka, and these annotations were used by Roary with an identity of $80 \%$ to identify the pangenome of strains, including 389 core genes, as previously described. ${ }^{12}$ The evolutionary history of studied strains was inferred using the maximum likelihood method, Kimura 2-parameter model within MEGA $7^{15}$, and iTOL (https://itol.embl.de/). This figure appears in color at www.ajtmh.org. 
was reported in an adult patient in South India with chronic liver disease. $^{12}$ Fewer than 10 cases of $V$. cholerae 01 bacteremia have been reported, including cases postdating a related outbreak and presenting with unusual features or complications. ${ }^{13,14}$

In addition to the spread of cholera through human movement, the role of asymptomatic long-term carriers and the role of gallbladder might be underestimated in the emergence of the disease.

Received December 2, 2019. Accepted for publication February 16, 2020.

Published online March 23, 2020.

Financial support: This study was supported by the Institut HospitaloUniversitaire (IHU) Méditerranée Infection, the National Research Agency under the program "Investissements d'avenir ", reference ANR-10-IAHU-03, the Région Provence Alpes Côte d'Azur, and European funding FEDER PRIMI.

Authors' addresses: Philippe Parola and Carole Eldin, Aix Marseille Univ, IRD, AP-HM, SSA, VITROME, Marseille, France, and IHUMéditerranée Infection, Marseille, France, E-mails: philippe.parola@ univ-amu.fr and pierre-edouard.fournier@univ-amu.fr. Hussein Anani, Aix Marseille Univ, IRD, AP-HM, SSA, VITROME, Marseille, France, E-mail: husseinanani2289@hotmail.com. Gregory Dubourg, JeanChristophe Lagier, Anthony Levasseur, and Didier Raoult, IHUMéditerranée Infection, Marseille, France, and Aix Marseille Univ, IRD, AP-HM, MEPHI, Marseille, France, E-mails: gregory.dubourg@aphm.fr, jclagier@yahoo.fr, anthony.levasseur@univ-amu.fr, and didier. raoult@gmail.com. Anthony Levasseur, Aix Marseille Univ, IRD, APHM, MEPHI, Marseille, France, E-mail: anthony.levasseur@univ-amu.fr. Pierre-Edouard Fournier, IHU-Méditerranée Infection, Marseille, France, E-mail: jean-paul.casalta@wanadoo.fr.

\section{REFERENCES}

1. Weil AA, Ryan ET, 2018. Cholera: recent updates. Curr Opin Infect Dis 31: 455-461.
2. WHO, 2018. Cholera Algeria. Diseases Outbreaks News. Available at: https://www.who.int/csr/don/14-september-2018-choleraalgeria/en/. Accessed September 14, 2018.

3. Boucher Y, 2016. Sustained local diversity of Vibrio cholerae 01 biotypes in a previously cholera-free country. mBio 7: e00570e00616.

4. Tarequl Islam M, Alam M, Boucher Y, 2017. Emergence, ecology and dispersal of the pandemic generating Vibrio cholerae lineage. Int Microbiol 20: 106-115.

5. Wallace C, 1976. Probable gallbladder infection in convalescent cholera patients. Lancet 289: 865-868.

6. Azurin JC, Kobari K, Barua D, Alvero M, Gomez CZ, Dizon JJ, Nakano El, Suplido R, Ledesma L, 1967. A long-term carrier of cholera: cholera Dolores. Bull World Health Organ 37: 745-749.

7. Gomez NA, Leon CJ, Gutierrez J, 1995. Acute acalculous cholecystitis due to Vibrio cholerae. Surg Endosc 9: 730-732.

8. Asnis DS, Golub R, Bresciani A, 1996. Vibrio cholerae 01 isolated in the gallbladder of a patient presenting with cholecystitis. Am J Gastroenterol 91: 2241-2242.

9. West BC, Silberman R, Otterson WN, 1998. Acalculous cholecystitis and septicemia caused by non-O1 Vibrio cholerae: first reported case and review of biliary infections with Vibrio cholerae. Diagn Microbiol Infect Dis 30: 187-191.

10. Nishikawa M, Hamanaka Y, Suzuki T, 1989. A case report of acute obstructive suppurative cholangitis in a non-0-1 Vibrio cholerae biliary carrier. Nihon Geka Hokan 58: 155-161.

11. Peterson EM, Jemison-Smith $P$, de la Maza LM, Miller D, 1982. Cholecystitis: its occurrence with cholelithiasis associated with a non-01 Vibrio cholerae. Arch Pathol Lab Med 106: 300-301.

12. Jesudason MV, Cherian AM, John TJ, 1993. Blood stream invasion by Vibrio cholerae 0139. Lancet 342: 431.

13. Gordon M, 2001. Three cases of bacteremia caused by Vibrio cholerae 01 in Blantyre, Malawi. Emerg Infect Dis 7: 1059-1061.

14. Jabeen K, Siddiqui J, Zafar A, Shakoor S, Ali N, Zaidi AKM, 2010. Vibrio cholerae 01 bacteremia in Pakistan: analysis of eight cases. Trans $R$ Soc Trop Med Hyg 104: 563-565.

15. Anani $H$, Abou Abdallah R, Chelkha N, Fontanini A, Ricaboni D, Mailhe M, Raoult D, Fournier P-E, 2019. Draft genome and description of Merdibacter massiliensis gen.nov., sp. nov., a new bacterium genus isolated from the human ileum. Sci Rep 9: 7931. 